Recanati/Miller Transplant Institute, Mount Sinai School of Medicine, New York, New York, USA

Address correspondence to: Jonathan S. Bromberg, Recanati/Miller Transplant Institute,

Mount Sinai School of Medicine, One Gustave L. Levy Place, Box 1104, New York, New York 10029, USA.

Phone: (212) 241-8938; Fax: (212) 348-2474; E-mail: jon.bromberg@mountsinai.org.

The field of allogeneic transplantation continues in search of its holy grail indefinite allograft survival and true alloantigen-specific tolerance without the need for chronic immunosuppression. That this goal has remained elusive reflects our fundamental uncertainty regarding the active and passive immunologic mechanisms that define the tolerant state. Diverse methods including costimulatory blockade, T-cell depletion, microand macrochimerism, and the induction of active, persistent regulatory mechanisms all improve allograft survival, but it is not known whether they lead to several distinct types of tolerance or whether they activate a single common pathway required for tolerance.

\section{Effects of costimulatory blockade}

Using the well-defined, functional rat renal transplant model, in which donor allografts are transplanted into MHCincompatible recipients, Waaga et al. in this issue of the JCI (1) induced tolerance in the recipients, using a short course of a blocking antibody to the costimulatory molecule CTLA4. They then subcloned and characterized T-cell lines derived either from acutely rejecting grafts, 7 days after transplantation, or from tolerant grafts, 100 days after transplantation. Interestingly, the rejecting allografts yielded CD4+ $\mathrm{T}$ cells with a Th1 phenotype, whereas Th2 type lines and clones were derived from the tolerated graft. These Th2 clones could suppress the characteristic activities of $T h 1$ clones seen in assays in vitro and in vivo, including their production of a distinctive set of cytokines and their ability to activate delayed type hypersensitivity. Importantly, the authors obtained human MHC class II, HLA-DR allopeptide-specific, CD4 ${ }^{+} \mathrm{T}$-cell clones generated from the peripheral blood of renal allograft recipients, and these human cell clones followed a similar pattern: Th1 type cells were generated from chronically rejecting patients; Th2 type cells and cytokines were derived from individuals with stable graft function.
Waaga and colleagues (1) suggest that the interplay of Th1 and regulatory $\mathrm{Th} 2$ cells are not only associated with, but also a necessary component for, indefinite graft survival and tolerance in the preclinical model, as well as for functional tolerance in the clinical situation. Indeed, these results are commensurate with earlier results presented by VanBuskirk et al. (2), who used a "trans vivo" delayed-type hypersensitivity assay, in which human cells are adoptively transferred into murine recipients and challenged with antigen. These authors worked with peripheral blood lymphocytes from renal and hepatic allograft recipients not receiving chronic immunosuppression, and they found that lymphocytes from recipients with stable graft function are hyporeactive to donorspecific alloantigen and manifest negative regulatory effects toward potentially responsive $\mathrm{T}$ cells when a specific alloantigen is encountered. These regulatory effects or suppression were dependent on TGF- $\beta$ or IL-10. It will be important to extend these observations to prove a causal role for the Th1 clones in initiating rejection and for the Th2 clones in preventing rejection.

\section{Cytokine profiles and tolerance: correlation or causation?}

The current report demonstrates an association between the presence of Th2 phenotypic clones and a tolerant state in rodents, as well as a similar association between Th2 cells and the ability of human transplant recipients to avoid chronic rejection. As Waaga et al. note (1), these findings are consistent with an influential paradigm, which holds that Th1-derived cytokines promote rejection and Th2-derived cytokines protect against this response by suppressing the delayed-type hypersensitivity reaction and by counteracting the actions of IFN- $\gamma$ on macrophages. In this model, the balance between cells of these two phenotypes determines the fate of the transplanted organ. Other data show, however, that the cytokine profile expressed does not always correlate with the outcome. Thus, both types of cytokines can be found in the rejecting organ, and, to date, neither group of cytokines has been found to be completely predictive of acute rejection. Furthermore, Matesic et al. demonstrated that allospecific $\mathrm{CD}^{+}$Th2 cells can mediate a delayed-type hypersensitivity response and that adoptive transfer of these cells can cause the rejection of allogeneic skin (3). Similarly, Kishimoto et al. (4) demonstrated that CTLA4Ig may successfully induce long-term engraftment and donor-specific tolerance even in STAT6-null recipients, which are deficient in Th2 responses. Lastly, knockout mice lacking the gene for the Th2 cytokine IL-4 are still able to achieve tolerance, thereby dispelling an oversimplified role of cytokines in rejection versus acceptance (5).

If the Th1/Th2 cytokine responses or ratios do not necessarily determine tolerance, the question remains whether other mechanisms may better define tolerogenic pathways. In the present report (1), tolerance was induced by costimulatory blockade with CTLA4Ig, a treatment that this group had previously shown to favor the predominant expression of Th2 cytokines (6). However, treatments that promote tolerance through costimulatory blockade do not always increase the relative expression of Th2 cytokines, but can affect the $T$ lymphocyte phenotype in diverse ways. Thus, treatment with anti-B7-1 $\mathrm{mAb}$ in vivo can skew autoreactive $T$ cells from a Th1 toward a Th2 phenotype, whereas blockade of B7-2 can generate a Th1 phenotype (7). B7 blockade can lead to selective death of activated Th1 cells stimulated with high-dose antigen or anti-CD3 mAb, but B7 blockade in the context of weaker T-cell stimulation can lead to the secretion of both Th1 and Th2 cytokines. In Th2-mediated diseases, costimulatory blockade with anti-CTLA4 $\mathrm{mAb}$ inhibits Th2 responses, while anti-B7-1 mAb leads to 
increased numbers of Th2 $\mathrm{CD}^{+}$regulatory cells, but both treatments inhibit the resultant disease process (7-9). Thus, the antigen presented, the pathway of presentation, the immunogenicity or affinity of the peptide, and the antigen load may all contribute to differences in the cytokine outcomes during tolerogenesis by costimulatory blockade. These observations raise the possibility that the Th2 predominance seen by Waaga et al. (1) could be an effect of, rather than the mechanism behind, the tolerant state induced by CTLA4Ig. Hence, some other aspect of costimulatory blockade may be responsible for tolerance.

\section{The diversity of regulatory immune cells}

These results now add to the growing body of data suggesting that multiple types of regulatory $T$ cells actively deviate or suppress the immune function of other $T$ cells to maintain tolerance and to control or prevent allograft rejection, xenograft rejection, autoimmunity, or hypersensitivity. The best-characterized of these cells is the CD4 $4^{+} \mathrm{CD} 25^{+} \mathrm{T}$-cell subset that inhibits autoimmune responses (10). Another regulatory $\mathrm{T}$ subset, the $\operatorname{Tr} 1$ cell, is derived from alloantigen-driven, human $\mathrm{CD}^{+}$cells stimulated in the presence of IL-10 (11). In addition, a $\mathrm{CD} 4^{+} \mathrm{V} \alpha 14$ natural killer $T$ cell has been described as important for the acceptance of xenografts (12). It remains unclear whether these cells are restricted to only certain classes or types of antigens, whether they act only early during the induction of tolerance, whether they are required for both shortterm and long-term maintenance of the tolerant state, and what effector mechanisms they employ. In addition, the relationships among these cells are not clear, since it is not known whether they represent different maturational states of the same population. Lastly, it is not known whether different approaches to immunosuppression result in the generation of similar or diverse regulatory cells. These remain challenges for future experimental tolerance studies.

The existence of diverse regulatory or suppressor $T$ cells suggests not only that immunosuppression influences the generation of these $T$ cells, but also that discrete types of antigen-presenting cells may be required for the induction of these regulatory $\mathrm{T}$ cells. Thus, recent reports suggest that immature dendritic cells (DCs) suppress the response to alloantigen or protein antigens and generate regulatory $\mathrm{T}$ cells $(13,14)$. Additional studies indicate that the elusive plasmacytoid DC may be the cell type responsible for generating suppressor or regulatory cells, the so-called DC2 (15, 16). Other specialized antigen-presenting cell types have also been described (17), and their role in tolerance to alloantigens or autoantigens remains speculative. How specialized DCs navigate lymphoid organs to come in contact with the precursors of regulatory $\mathrm{T}$ cells to generate suppressors is also unknown. As for Tcell subsets, similar issues for DC cell subsets remain to be explored, including the classes of antigens, the influences that induce or maintain tolerance, the relationship among various DC populations, and their interaction with pharmacologic immunosuppressants.

\section{Outlook}

Like all good research, the current report (1) suggests many avenues for future investigations. As noted above, DCs important for the generation of regulatory $\mathrm{T}$ cells, the enumeration of regulatory $T$-cell subsets, the interrelationship of these subsets, the role of costimulatory blockade, and the nature of Th2 effects on graft survival are important issues that must be elucidated. From a clinical perspective, the diverse outcomes of costimulatory blockade suggest that this approach may not offer a panacea for graft rejection. To develop additional therapies, it will be important to learn how different types of pharmacological immunosuppression affect the development of regulatory T-cell and DC subsets. In addition, it is clear that "96-well plate immunology" does not substitute for the physical domains and microanatomic interactions that orchestrate the interactions among $\mathrm{T}$ cells and DCs in secondary lymphoid organs. From the study of diverse methods of tolerization, several overarching principles have emerged: the cross-regulation of immune cell subsets, the central role of chemokines and their receptors (18-20), and the significance of secondary lymphoid organ structure (21). Further work building on these insights may bring to light a final common pathway that leads to immunosuppression and tolerance.

1. Waaga, A.M., et al. 2001. Regulatory functions of self-restricted MHC class II allopeptide-specific
Th2 clones in vivo. J. Clin. Invest. 107:909-916. 2. VanBuskirk, A.M., et al. 2000. Human allograft acceptance is associated with immune regulation. J. Clin. Invest. 106:145-155.

3. Matesic, D., et al. 1998. Type 2 immune deviation has differential effects on alloreactive $\mathrm{CD}^{+}$and $\mathrm{CD8}^{+} \mathrm{T}$ cells. J. Immunol. 161:5236-5244.

4. Kishimoto, K., et al. 2000. The role of CD154CD40 versus CD28-B7 costimulatory pathways in regulating allogeneic Th 1 and Th2 responses in vivo. J. Clin. Invest. 106:63-72.

5. Lakkis, F. 1998. Role of cytokines in transplantation tolerance: lessons learned from gene-knockout mice. J. Am. Soc. Nephrol. 9:2361-2367.

6. Sayegh, M.H., et al. 1995. CD28-B7 blockade after alloantigenic challenge in vivo inhibits Th1 cytokines but spares Th2. J. Exp. Med. 181:1869-1874.

7. Anderson, D.E., Sharpe, A.H., and Hafler, D.A 1999. The B7-CD28/CTLA4 costimulatory path ways in autoimmune disease of the central nerv ous system. Curr. Opin. Immunol. 11:677-683.

8. Xu, H., Heeger, P.S., and Fairchild, R.L. 1997. Distinct rolls for B7-1 and B7-2 determinants during priming of effector $\mathrm{CD}^{+} \mathrm{Tcl}$ and regulatory $\mathrm{CD}^{+} \mathrm{Th} 2$ cells for contact hypersensitivity. $J$ Immunol. 159:4217-4226.

9. Sakurai, J., et al. 2000. Blockade of CTLA-4 signals inhibits Th 2 mediated murine chronic graftversus-host disease by enhanced expansion of regulatory CD8 T cells. J. Immunol. 164:664-669.

10. Read, S., Malmstrom, V., and Powrie, F. 2000. Cytotoxic $\mathrm{T}$ lymphocyte-associated antigen 4 plays an essential role in the function of $\mathrm{CD} 25\left(^{+}\right)$ $\mathrm{CD} 4\left(^{+}\right)$regulatory cells that control intestinal inflammation. J. Exp. Med. 192:295-302.

11. Groux, H., et al. 1997. A CD4 ${ }^{+}$T-cell subset inhibits antigen-specific T-cell responses and prevents colitis. Nature. 389:737-742.

12. Ikehara, Y., et al. 2000. CD4 $4^{+}$V $\alpha 14$ natural killer $\mathrm{T}$ cells are essential for acceptance of rat islet xenografts in mice. J. Clin. Invest. 105:1761-1767.

13. Jonuleit, J., Schmitt, E., Schuler, G., Knop, J., and Enk, A.H. 2000. Induction of interleukin 10-producing, nonproliferating $\mathrm{CD} 4^{+} \mathrm{T}$ cells with regulatory properties by repetitive stimulation with allogenic immature human dendritic cells. J. Exp. Med. 192:1213-1222.

14. Dhodapkar, M.V., Steinman, R.M., Krasovsky, J., Munz, C., and Bhardwaj, N. 2001. Antigen-specific inhibition of effector $\mathrm{T}$ cell function in humans after injection of immature dendritic cells. J. Exp. Med. 193:233-238.

15. Blom, B., Ho, S., Antonenko, S., and Liu, Y.J. 2000 Generation of interferon $\alpha$-producing pre-dendritic cell (DC) 2 from human CD $34^{+}$hematopoietic stem cells. J. Exp. Med. 192:1785-1795.

16. Spits, H., Couwenberg, F., Bakker, A.Q., Weijer, K. and Uittenbogaart, C.H. 2000. Id 2 and Id 3 inhibit development of $\mathrm{CD} 34^{+}$stem cells into preden dritic cell (pre-DC)2 but not into pre-DC1: evidence for a lymphoid origin of pre-DC2. J. Exp. Med. 192:1775-1783.

17. Limmer, A., et al. 2000. Efficient presentation of exogenous antigen by liver endothelial cells to $\mathrm{CD}^{+} \mathrm{T}$ cells results in antigen-specific T-cell tolerance. Nat. Med. 6:1348-1354.

18. Robinson, L.A., et al. 2000. A role for fractalkine and its receptor $\left(\mathrm{CX}_{3} \mathrm{CR} 1\right)$ in cardiac allograft rejection. J. Immunol. 165:6067-6072.

19. Gao, W., et al. 2000. Targeting of the chemokine receptor CCR1 suppresses development of acute and chronic cardiac allograft rejection. J. Clin. Invest. 105:35-44.

20. Hancock, W.W., et al. 2000. Requirement of the chemokine receptor CXCR3 for acute allograft rejection. 192:1515-1519.

21. Lakkis, F.G., Arakelov, A., Konieczny, B.T., and Inoue, Y. 2000. Immunologic 'ignorance' of vascularized organ transplants in the absence of secondary lymphoid tissue. Nat. Med. 6:686-688. 